\title{
Desempenho do modelo estocástico de média-variância para o mercado brasileiro de ações
}

\author{
Henrique Helfer Hoeltgebaum \\ Departamento de Estatística UFRGS \\ hhhelfer@hotmail.com

\section{Tiago Pascoal Filomena} \\ Programa de Pós-Graduação em Administração de Empresas, PPGA-UFRGS \\ tpfilomena@ea.ufrgs.br

\section{Denis Borenstein} \\ Programa de Pós-Graduação em Administração de Empresas, PPGA-UFRGS \\ dborenstein@ea.ufrgs.br
}

\author{
Miguel Lejeune \\ Escola de Administração, George Washington University \\ mlejeune@gwu.edu
}

Flavio Augusto Ziegelmann

Departamento de Estatística, UFRGS; Programa de Pós-Graduação em Economia,PPGE-UFRGS;Programa de Pós-Graduação em Administração de Empresas, PPGA-UFRGS

flavioaz@mat.ufrgs.br

\section{RESUMO}

Os modelos de média-variância deotimização de carteira apresentam questionamentos em relação ao seu efetivo desempenho devido ao chamado erro de estimação.Em consequência, a otimização estocástica vem aumentando sua importância pois permite à possibilidade da inclusão da incerteza na estimativa dos parâmetros. Neste estudo foi avaliado o desempenho do modelo de otimização de carteira proposto por Bonami e Lejeune (2009), e reformulado por Filomena e Lejeune (2012), no mercado brasileiro de ações. Ambos os modelos podem ser caracterizados como versões probabilísticas do modelo clássico de média-variância proposto por Markowitz (1952). O modelo de Filomena e Lejeune (2012) apresentou desempenho médio superior aos benchmarks IBRX-50 e IBOVESPA. No entanto, o mesmo apresentou resultados viáveis em apenas $12,5 \%$ dos 64 cenários de teste. Embora isto possa ser considerado uma limitação do modelo,também pode ser analisado como um benefício para os investidores, pois oferece pontos de entrada e saída do mercado de ações.

Palavras-chave: Estocástico, Carteira, Média-variância.

\begin{abstract}
The estimation error is a well-documented problem of the mean-variance optimization models. Therefore, stochastic optimization has increased its importance on portfolio optimization given its ability to include uncertainty as a parameter. In this study, the performance of the model proposed by Filomenaand Lejeune (2012), a reformulated version of a previous model developed by Bonami and Lejeune (2009), was evaluated for the Brazilian stock market. Both models can be characterized as probabilistic versions of the classical model developed by Markowitz (1952). The performance of the model was superior when compared to the market benchmarks IBRX-50 and IBOVESPA. However, it presented feasibility in just $12,5 \%$ of the 64 test cases. Although this can initially be seen as a strong limitation for the practical use of the model, this can be considered as a very important pragmatic information for investors, since the model provides possible entry and exit points on the stock market.
\end{abstract}

Keywords: Stochastic, Portfolio, Mean-variance. 


\section{Introdução}

Os modelos de otimização de carteira de investimento têm recebido atenção desde o modelo clássico de médiavariância proposto por Markowitz (1952). Este modelo propõe uma formulação matemática para o problema de seleção de um portfólio de ativos de modo que o investidor leve em consideração o "trade-off" entre retorno e risco (CORNUEJÓLS; TÜTÜNCÜ, 2007). Segundo Zenios (2008), a análise de média-variância estuda o desempenho de portfólios em um espaço de retorno versus risco, onde o retorno é medido pela taxa de retorno esperado do portfólio, e o risco é medido pela variância da taxa de retorno do mesmo. O principal objetivo desta análise é formar portfólios eficientes de ativos com mínimo risco e que garantam um retorno mínimo requerido denominado de "target". Um portfólio é denominado eficiente se este tem um retorno esperado máximo entre todos os portfólios formados com a mesma variância, ou, alternativamente, se possuia menor variância entre todos os portfólios que apresentam um determinado nível mínimo de retorno esperado (CORNUEJÓLS; TÜTÜNCÜ, 2007). O conjunto de portfólios eficientes para dado nível de risco forma a fronteira eficiente, a qual é obtida ao se resolver diversas vezes o problema de média-variância para diferentes níveis de retorno alvo.

O modelo clássico de Markowitz, denominado aqui de $M K W$, continua sendo amplamente discutido na literatura (CORAZZA; FAVARETTO, 2007; CORNUEJÓLS; TÜTÜNCÜ, 2007; DENTCHEVA; RUSZCZYNSKI, 2006; LI et al., 2006; SCHERER; MARTIN, 2005). Uma das críticas é que o modelo concentra muito a alocação em alguns ativos no portfólio. Outra crítica frequente ao modelo é que este considera que o retorno esperado dos ativos e a matriz de variância-covariância são perfeitamente conhecidos, tratando-os como parâmetros determinísticos. Adicionalmente, os retornos médios dos ativos são considerados como os verdadeiros retornos esperados. Porém obter estimativas precisas destes parâmetros é algo complexo nos mercados financeiros. Existem inúmeras fontes de erro que afetam essa estimação, conduzindo ao chamado risco de estimação em seleção de carteira (BAWA et al., 1979). De fato, o risco de estimação tem se mostrado um fator de decisões errôneas, pois como assinalaram vários estudos empíricos (BROADIE, 1993; CERIA; STUBBS, 2006; MERTON, 1980; CHOPRA; ZIEMBA, 1993), o modelo clássico de Markowitz é extremamente sensível à estimação dos retornos dos ativos, mais inclusive do que à estimação da matriz de variância-covariância. Ou seja, pequenas variações na estimativa do retorno esperado dos ativos podem levar a uma grande reformulação do portfólio com ativos distintos e a uma relação de grande rotatividade de ativos ao longo dos períodos (CERIA; STUBBS, 2006; CORNUEJÓLS; TÜTÜNCÜ, 2007).
Portanto, existe a necessidade de formular um modelo que não seja afetado por pequenas perturbações nos momentos do retorno estimado e nos estados do mercado, pois a incerteza existente no mercado e a instabilidade do modelo $M K W$ dificultam sua utilização na prática (TÜTÜNCÜ; KOENIG, 2004). Uma nova formulação do modelo clássico de $M K W$, que considere a incerteza associada com a estimação do retorno esperado dos ativos, é necessária. Para isso, inicialmente assume-se que o estimador para o retorno esperado é estocástico e caracterizado por uma distribuição de probabilidade qualquer na qual são considerados o primeiro e o segundo momentos. Visto que existe uma probabilidade de se obter um retorno esperado extremo em séries financeiras, não há garantia de que estes sejam normalmente distribuídos. Este trabalho tem como principal objetivo aplicar ao mercado brasileiro de ações a versão probabilística do modelo de $M K W$ proposto por Bonami e Lejeune (2009), e reformulado por Filomena e Lejeune (2012). Este modelo apresenta como principal atributos a desnecessidade dos retornos serem normalmente distribuídos. O conhecimento incompleto do comportamento do retorno de um ativo é considerado pela representação de seu retorno médio como uma variável aleatória, atrelando assim a incerteza na estimação do retorno esperado dos ativos. Em consequência, obtém-se a diminuição da sensibilidade do portfólio à estimativa do retorno esperado.

O presente trabalho foca na validação do modelo alternativo formulado por Filomena e Lejeune (2012). É dada ênfase a incerteza atrelada à estimação do retorno esperado, assumindo-se que esta seja estocástica e caracterizada por uma distribuição de probabilidade, e não somente pelo primeiro momento de uma distribuição normal. O modelo alternativo ao $M K W$ proposto por Bonami e Lejeune (2009) será denominado de Portfólio Estocástico $(P E)$ e é constituído substituindo a restrição clássica que garante o retorno da carteira por uma restrição probabilística que garante o retorno da carteira com certa probabilidade $p$, adicionando-se assim aleatoriedade ao modelo clássico de $M K W$. Ao adicionarmos essa restrição, o modelo $P E$ pode ser classificado como um modelo de programação estocástica com restrição probabilística, os quais são, em geral, mais complexos numericamente que os modelos de programação matemática determinística (como o modelo clássico de $M K W$ ). Neste tipo de problema, em geral, é necessária a derivação de um determinístico equivalente para que este possa ser resolvido numericamente. Filomena e Lejeune (2012) reformularam o modelo PER, incorporando esta habilidade. Este modelo será denominado de Portfólio Estocástico Reformulado $(P E R)$. Maiores detalhes deste modelo serão discutidos na Seção 3. 
A principal contribuição deste trabalho é a de avaliar o desempenho do modelo PER junto ao mercado brasileiro de ações, definindo o potencial de sua prática utilização a este mercado, caracterizado por um número pequeno de ativos com alta liquidez e alta volatilidade. Também é estudada a estratégia de assumir que a média da distribuição de probabilidade dos retornos é um bom estimador para o retorno futuro, ou seja, pela aplicação direta do modelo $M K W$. Para o processo de avaliação dos dois modelos serão utilizados os dados de fechamento semanal dos ativos que constaram no IBRX-50 na data de 23 de Agosto de 2011. Essas avaliações serão posteriormente detalhadas na Seção 4, mas em essência, os modelos de otimização de portfólio no mercado brasileiro de ações serão avaliados a partir de 3 métricas (retorno, volatilidade e índice Sharpe) e comparados com os benchmarks de mercado, IBRX-50 e IBOVESPA.

Os resultados do modelo PER foram promissores e o classificam como uma ferramenta gerencial de portfólio para médio/longo prazo, visto que este encontra bons resultados quando estimado para 12 e 24 semanas. A média dos retornos é superior ao modelo $M K W$, bem como aos benchmarks utilizados. A média dos retornos ajustados ao risco (índice Sharpe) do modelo PER, em geral, foram superiores ao mesmo índice dos benchmarks de mercado, e inferiores aos apresentados pelo modelo $M K W$. Analisando-se os portfólios obtidos pelos dois modelos, constata-se que o modelo $M K W$ concentra os investimentos em poucos ativos, o que não ocorre com o modelo PER. No entanto, o modelo PER apresenta certas limitações, apresentando respostas viáveis em apenas $12,5 \%$ dos casos em que fora utilizado.Paradoxalmente, este fato pode ser interpretado positivamente pelos investidores, pois oferece uma indicação de pontos de entrada e saída do mercado de ações. O artigo está organizado como se segue. Na próxima seção é introduzido o modelo PER e, em seguida, sua aproximação determinística. A aplicação no mercado brasileiro é apresentada na seção 4. As questões de desempenho dos modelos, diversificação de portfólio e particularidades do modelo PER são apresentadas e discutidas na seção 4. A seção 5 apresenta as conclusões deste trabalho.

\section{Modelagem}

O modelo clássico de média-variância de Markowitz pode ser descrito de várias maneiras (CORNUEJÓLS; TÜTÜNCÜ, 2007). No presente trabalho será utilizada a seguinte formulação, a qual será definida como modelo $M K W$,

$$
\begin{aligned}
& M K W: \min \quad w^{T} £ w \\
& S t \quad \mu^{T} w \geq R \\
& \sum_{j=1}^{n} w_{j}=1 \\
& w_{j} \geq 0, j=1, \ldots, n
\end{aligned}
$$

onde $n$ representa o número de ativos existentes no mercado, $R$ é o retorno mínimo requerido (target), $\mathrm{w}_{\mathrm{j}}$ é o peso do ativo $j$ na carteira, $\Sigma$ é a matriz de variânciacovariância estimada $\mathrm{e} \mu^{\mathrm{T}}$ é um vetor transposto composto pelas estimativas dos retornos esperados dos $n$ ativos. No modelo $M K W$ os retornos médios são tomados como os verdadeiros retornos esperados sem que haja qualquer penalidade atrelada. A função objetivo do modelo busca minimizar a variância do portfólio, composto pelos ativos selecionados, com a garantia de que se obtenha um retorno mínimo requerido.

O modelo alternativo proposto pode ser formulado conforme segue:

$$
\begin{aligned}
& P E: \min w^{T} \Sigma w \\
& \text { St } \quad \mathbb{P}\left(\sum_{j=1}^{n} \xi_{j} w_{j} \geq R\right) \geq p
\end{aligned}
$$

onde $\xi$ é um vetor aleatório composto pelas estimativas dos retornos esperados dos $n$ ativos, $p$ é a probabilidade com que o retorno $R$ seja atendido. $\mathrm{O}$ vetor de médias e a matriz de variância-covariância são definidos como: $\mu=\left(\mu_{1}, \ldots, \mu_{n}\right)^{T}$ onde $\mu_{j}=E\left(\xi_{j}\right) \mathrm{e}$ $\Sigma=E\left[(\xi-\mu)(\xi-\mu)^{\mathrm{T}}\right] \quad$ para $j=1, \ldots, n$.

O modelo $P E$, e, consequentemente, o modelo PER (que será descrito na Seção 3) pertencem à família de modelos de risco downside, que objetivam evitar que o retorno caia abaixo de um determinado valor aceitável. A medida de risco considerada neste estudo é relacionada ao critério de safety-first de Roy (1952) que identifica o portfólio ótimo para o qual a probabilidade do seu retorno cair abaixo de um limite mínimo aceitável é minimizada. A medida de risco de Roy é próxima do índice Sharpe (BONAMI; LEJEUNE, 2009), o qual maximiza a relação entre retorno médio e risco do 
portfólio. O índice Sharpe (SHARPE, 1966) caracteriza o quão bem o retorno de um portfólio compensa o investidor pelo risco assumido. Quanto maior o número do índice, melhor é o indicativo para o investidor. No presente trabalho utiliza-se um índice Sharpe alterado sem o ativo livre de risco, pois: (i) foram disponibilizados apenas ativos de risco na carteira; e (ii) o período de estudo inclui duas grandes crises (Imobiliária/2008 e Dívida Externa/2011) que somadas ao alto valor da taxa livre de risco brasileira acabam gerando índices Sharpe quase que invariavelmente negativos. Portanto, definimos o índice Sharpe como:

$$
S R=\frac{\text { retorno realizado }}{\text { volatilidaderealizada }}
$$

Infelizmente, o modelo $P E$ é de difícil solução, pois apresenta restrições estocásticas. A seguirserá apresentada uma reformulação do modelo $P E$ para que este possa ser resolvido numericamente.

\section{Aproximação Determinística}

Segundo Filomena e Lejeune (2012) a aleatoriedade do retorno estimado dos ativos leva à existência de uma restrição probabilística descrita em (5). Devido à inclusão dessa restrição, o problema $P E$ é classificado como um modelo de programação estocástica, de difícil solução. Nessa classe de problemas, em geral, derivase um determinístico equivalente para que este possa ser resolvido numericamente. As provas matemáticas dos resultados desta seção não serão apresentadas; as mesmas são encontradas em Bonami e Lejeune (2009). O equivalente determinístico da restrição probabilística é o seguinte termo:

$$
\mu^{\mathrm{T}} \mathrm{w}+\mathrm{F}_{(\mathrm{w})}^{-1}(1-\mathrm{p}) \sqrt{\mathrm{w}^{\mathrm{T}} \sum \mathrm{w}} \geq \mathrm{R}
$$

onde $F$ é a distribuição de probabilidade acumulada do retorno da carteira padronizado (média zero e desvio padrão igual a um), e $\mathrm{F}_{(\mathrm{w})}^{-1}$ a sua inversa. Com base na equação acima, buscam-se distribuições de probabilidades que tornem esta restrição cônica de segunda ordem (second-orderconic). Segue do teorema apresentado em Bonami e Lejeune (2009) que se $\Sigma$ for uma matriz positiva semidefinida e se a distribuição de probabilidade de $\xi_{\mathrm{j}} \mathrm{w}_{\mathrm{j}}$ for simétrica ou tiver curtose positiva, $\mathrm{F}_{(\mathrm{w})}^{-1}(1-\mathrm{p})$ será um número $k$ não negativo, e o equivalente determinístico da restrição probabilística apresentado acima pode ser formulada como a seguinte restrição cônica de segunda ordem,

$$
\mu^{\mathrm{T}} \mathrm{W}-\mathrm{K} * \sqrt{\mathrm{w}^{\mathrm{T}} \sum \mathrm{w}} \geq \mathrm{R},
$$

Quando a distribuição de probabilidades é caracterizada pelos seus dois primeiros momentos, ou seja, a média e a variância, a constante $k$ pode ser aproximada por (BONAMI; LEJEUNE, 2009)

$$
k=\sqrt{\frac{p}{1-p}} .
$$

Sendo assim o modelo PE tomaria a seguinte forma, denominada de Portfólio Estocástico Reformulado (PER),

$$
\begin{gathered}
\text { PER: } \min w^{T} \Sigma w \\
\text { Sujeito } \grave{a} \quad \mu^{\mathrm{T}} \mathrm{w}-\sqrt{\frac{p}{1-p}} * \sqrt{\mathrm{w}^{\mathrm{T}} \Sigma \mathrm{w}} \geq \mathrm{R}
\end{gathered}
$$

$$
\text { (3), (4) }
$$

A desigualdade presente na nova restrição (6) destaca o fato de que o retorno esperado do portfólio necessário para ultrapassar o patamar de retorno target é aumentado por um termo de penalidade

$$
\sqrt{\frac{p}{1-p}} * \sqrt{w^{T} \Sigma w}
$$

Desta forma, o portfólio deve ter um desempenho de

$$
R+\sqrt{\frac{p}{1-p}} * \sqrt{w^{T} \sum w}
$$

para que o retorno target seja atingido com certa probabilidade $p$. Isto é um contraste com o clássico Markowitz, no qual a carteira apenas deve atingir um desempenho $R$. Caso se atribua um número que tenda a zero para $p$, estaremos novamente diante do problema $M K W$, onde o termo de penalidade apresentado acima tende a zero.

Uma das necessidades básicas para otimização deste problema é que a matriz $\Sigma$ seja positiva semi-definida, propriedade que é facilmente violada quando se tenta otimizar simultaneamente o portfólio com um elevado número de ativos. Filomena e Lejeune (2012) propuseram uma reformulação do problema que relaxa essa propriedade e ainda aumenta significativamente o desempenho computacional do problema (FILOMENA; LEJEUNE, 2011; 2012). 


\section{Experimentação Computacional}

Nesta seção serão apresentadas as avaliações realizadas com os modelos PER e $M K W$ no mercado brasileiro. Descrições detalhadas dos dados, das análises e dos resultados seguem abaixo.

\subsection{Coleta dos Dados}

Para a coleta dos dados utilizou-se o software Economática, onde se obtiveram os preços de fechamento semanal das ações negociadas na Bovespa. O preço é ajustado para dividendos e stocks split, em um período abrangendo de 01 de janeiro de 2007 a 26 de agosto de 2011. Além disso, com o objetivo de selecionar as ações mais líquidas no mercado brasileiro, foram utilizadas apenas as ações que constavam no IBRX-50 na data de 23 de agosto de 2011. Sendo assim, a amostra se restringiu aos preços de fechamento semanal de 50 ativos entre 05 de janeiro de 2007 e 26 de agosto de 2011.

\subsection{Avaliações no mercado brasileiro de ações}

Para a realização das avaliações no mercado, os fechamentos semanais do banco de dados são divididos em períodos, os quais são compostos por 52 semanas, ou seja, 1 ano. Note-se que os ativos que abriram capital posteriormente ao ano de referência são utilizados apenas no ano seguinte. Desta forma, para o ano de 2007 são disponibilizados 38 ativos para otimização; em 2008, 43; em 2009, 47 e em 2010 e 2011, 50, respectivamente. Para a otimização são utilizados 243 períodos no total. Os testes foram desenvolvidos considerando o seguinte projeto de experimentos:

1. Inicialmente, selecionam-se os preços do primeiro período, que abrangem o intervalo de 5 de janeiro de 2007 até 28 de dezembro de 2007.

2. Com a definição do período de análise calcula-se os problemas de otimização $P E R$ e $M K W$.

3. São utilizados os preços de 12 e 24 semanas posteriores ao período de otimização dos ativos que constam no portfólio formado pela otimização no passo 2 . As ponderações indicadas pela otimização no passo 2 são aplicadas aos respectivos ativos, com a finalidade de prever o desempenho do portfólio em 12 e 24 semanas posteriores ao período de otimização. A partir disso, geram-se os três indicadores para avaliar o desempenho do portfólio formado pelos modelos $P E R, M K W$, IBRX50 e do IBOVESPA: retorno, volatilidade (ou risco) e índice Sharpe. No exemplo, faz-se uso do período da semana 1 a 52, sendo assim aplicam-se as ponderações nos ativos indicadas neste período nos preços das semanas 52 a 64 e 52 a 76.
Após finalizar o processo descrito acima, passam-se 3 períodos sem análise e repete-se o processo novamente. Ou seja, após analisar o período de 1-52, analisa-se o período de 4-55 e assim analogamente até o período 190-241, que ao final compõem 64 períodos.

As avaliações são realizadas em períodos de tempo que se sobrepõem, para que o modelo seja colocado à prova o maior número de vezes, procurando captar seu comportamento sob o maior número de cenários possíveis ocorridos durante esses períodos (crises, altas de mercado ou mercado estável).

\subsection{Discussão dos resultados}

O objetivo principal das avaliações é verificar as características dos portfólios formados em termos práticos, assumindo o ponto de vista de um investidor. São comparados os resultados do PER e do $M K W$ a benchmarks de mercado (IBRX-50 e IBOVESPA) a partir das três métricas de desempenho. Inicialmente, faz-se uma análise do comportamento do parâmetro $p$ sob o comportamento do modelo; a seguir, discutem-se os portfólios formados pelos modelos; e, finalmente, apresenta-se interpretações dos resultados obtidos pelos modelos.

Sabe-se a priori que o mercado brasileiro de ações tem uma volatilidade mais alta do que o mercado norte-americano. Dada essa informação, ao utiliza-se o modelo $P E R$, deve-se atentar para o fato de que, a definição de uma probabilidade $p$ muito alta para atingir o retorno target poderá gerar uma resposta inviável para o modelo, isto é, resultando em inviabilidade na solução pela restrição exagerado do espaço de soluções. Será definido no presente trabalho um $p$ de 0,2 e um retorno target fixado de no mínimo $20 \%$ ao ano, o que equivale a um rendimento de $0,35 \%$ semanal. Portanto, admite-se que exista uma probabilidade de $20 \%$ de que o retorno almejado de $0,35 \%$ semanal seja atendido.

Com estes parâmetros, o PER obteve apenas8 repostas viáveis nos 64 períodos testados. Porém, deve-se notar que se trata do mercado de ações, o qual vem enfrentando nos últimos anos duas grandes crises (Crise Imobiliária 2008-2009 e Crise das dívidas Soberanas 2010-2011), e que estamos disponibilizando para a formação do portfólio apenas ativos de risco. Considerando estes cenários macroeconômicos, é improvável obter um retorno semanal maior ou igual a 0,35\%. A situação piora se considerarmos à adição da penalidade definida em relação ao $p$. Desta forma, o modelo só obteve soluções em situações de grande alta no mercado, o que ocorre nos oito períodos em que a resposta do modelo foi viável. Deve-se salientar que o número de soluções viáveis poderia ser aumentado com a diminuição do valor de $p$. Além disto, soluções não viáveis possuem uma interpretação interessante sobre o 
ponto de vista de um investidor, caracterizando pontos de entrada e saída do mercado. A Figura 1 evidencia a grande alavancagem nos pontos do IBOVESPA nos períodos nos quais o modelo PER obteve resultados viáveis. O IBOVESPA subiu de 36.595 mil pontos em 28 de Novembro de 2008 (período 100) a $69.421 \mathrm{mil} \mathrm{em}$ 16 de Abril de 2010 (período 172), o que representou uma alta de $89,70 \%$ do índice no período.

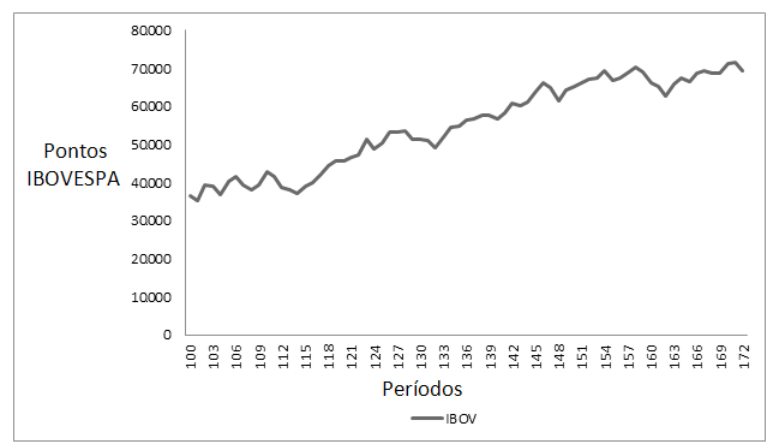

Figura 1 - Pontos do IBOVESPA entre 28 de Novembro de 2008 a 16 de Abril de 2010.

A Tabela 1 apresenta uma análise da influência de $R$ e $P e$ no retorno médio do portfólio. Os resultados dos oito períodos testados indicam o quão "caro" é almejar um retorno target da magnitude estabelecida acima, visto que atrelado a este existe uma constante de penalidade que infla essa quantidade conforme a restrição

$R+\sqrt{\frac{p}{1-p}} * \sqrt{w^{T} \Sigma w}$, levando a resposta do modelo a ser inviável. Considerando a quantidade $P e=\sqrt{\frac{p}{1-p}} * \sqrt{w^{T} \Sigma w}$

como um termo de penalidade sobre o retorno requerido, tem-se que o retorno semanal é o retorno médio obtido com o portfólio selecionado pelo modelo PER. Pode-se concluir que a medida que o retorno diminui, a proporção da constante de penalidade também é diminuída. Um exemplo é o fato que ocorre no período 109-160, que corresponde respectivamente a 30 de janeiro de 2009 a 22 de janeiro de 2010, que tem a menor constante de penalidade atrelada, representando 2,6 vezes a proporção de retorno. Assim, o penalty semanal fica em $0,91 \%$, que somado a $0,35 \%$ do retorno target semanal, o portfólio deve desempenhar um retorno médio de 1,26\%. Em síntese, deve-se atentar para essa possível questão de um trade-off entre o retorno target do portfólio e a probabilidade de obter este retorno, pois, caso se deseje um retorno elevado com uma probabilidade alta de ser atingido, a constante de penalidade também será inflada e possivelmente o problema não será solucionado.

Tabela 1 - Retorno semanal e as respectivas proporções de penalidade e retorno almejado.

\begin{tabular}{|c|c|c|c|c|c|c|c|c|}
\hline Período & $\mathbf{1 0 0 - 1 5 1}$ & $\mathbf{1 0 3 - 1 5 4}$ & $\mathbf{1 0 6 - 1 5 7}$ & $\mathbf{1 0 9 - 1 6 0}$ & $\mathbf{1 1 2 - 1 6 3}$ & $\mathbf{1 1 5 - 1 6 6}$ & $\mathbf{1 1 8 - 1 6 9}$ & $\mathbf{1 2 1 - 1 7 2}$ \\
\hline $\begin{array}{c}\text { Retorno } \\
\text { semanal }\end{array}$ & $1,65 \%$ & $1,46 \%$ & $1,30 \%$ & $1,26 \%$ & $1,34 \%$ & $1,30 \%$ & $1,39 \%$ & $1,46 \%$ \\
\hline $\mathbf{R}$ & $21 \%$ & $24 \%$ & $27 \%$ & $28 \%$ & $26 \%$ & $27 \%$ & $25 \%$ & $24 \%$ \\
\hline $\mathbf{P e}$ & $79 \%$ & $76 \%$ & $73 \%$ & $72 \%$ & $74 \%$ & $73 \%$ & $75 \%$ & $76 \%$ \\
\hline
\end{tabular}

A Tabela 2 mostra os portfólios formados, com a respectiva participação dos ativos, formados pelo modelo $P E R$ nos oito períodos em que este encontra uma resposta viável. Pelos resultados obtidos com o modelo PER, observa-se que os portfólios formados por este ao longo desses oito períodos contemplam a questão da diversificação de setores. O portfólio formado é composto por empresas do setor telefônico, construtoras, de aviação, entre outras. O nome das empresas foram omitidos e substituídos por nomes genéricos. Segundo Luenberger (1998), portfólios com apenas poucos ativos podem estar sujeitos a um risco maior. A diversificação funciona como uma proteção para os investidores, visto que formando portfólios diversificados, diminui-se a exposição ao risco.

É possível analisar a participação de cada ativo nos portfólios formados pelo modelo. $\mathrm{O}$ ativo 1, por exemplo, está presente nos 8 portfólios e tem a maior média de participação nos 8 períodos, como pode ser observado pela última coluna. Já o ativo 13 obtém a menor média de participação, visto que este é selecionado apenas no portfólio formado no primeiro período. Embora em média não existam concentrações excessivas de ativos, todas inferiores a $20 \%$, algumas concentrações podem ser questionadas por investidores, pois em alguns casos há concentrações superiores a $20 \%$. 
Tabela 2 - Portfólios formados e as respectivas participações dos ativos pelo modelo PER.

\begin{tabular}{|c|c|c|c|c|c|c|c|c|c|}
\hline $\begin{array}{c}\text { Cód. } \\
\text { Ativo }\end{array}$ & $\mathrm{P} 1$ & $\mathrm{P} 2$ & $\mathrm{P} 3$ & $\mathrm{P} 4$ & $\mathrm{P} 5$ & $\mathrm{P} 6$ & $\mathrm{P} 7$ & $\mathrm{P} 8$ & Média \\
\hline 1 & $6,8 \%$ & $18,9 \%$ & $21,4 \%$ & $20,9 \%$ & $25,4 \%$ & $17,1 \%$ & $13,6 \%$ & $14,8 \%$ & $17,4 \%$ \\
\hline 2 & $4,4 \%$ & $3,0 \%$ & & & & & & & $3,7 \%$ \\
\hline 3 & & & $1,0 \%$ & $1,4 \%$ & $1,4 \%$ & $3,7 \%$ & $1,1 \%$ & $3,0 \%$ & $1,9 \%$ \\
\hline 4 & $6,8 \%$ & $6,4 \%$ & $4,3 \%$ & $8,9 \%$ & $3,4 \%$ & $3,4 \%$ & $5,2 \%$ & $3,2 \%$ & $5,2 \%$ \\
\hline 5 & & $8,2 \%$ & & $0,9 \%$ & $1,1 \%$ & & & & $3,4 \%$ \\
\hline 6 & $6,1 \%$ & $6,5 \%$ & $0,4 \%$ & $4,2 \%$ & $4,4 \%$ & $7,7 \%$ & $7,8 \%$ & $9,4 \%$ & $5,8 \%$ \\
\hline 7 & $15,1 \%$ & $15,9 \%$ & $8,5 \%$ & $9,2 \%$ & $9,1 \%$ & $0,1 \%$ & & & $9,7 \%$ \\
\hline 8 & $9,3 \%$ & $3,8 \%$ & $3,1 \%$ & $1,6 \%$ & $1,3 \%$ & $1,6 \%$ & & $2,6 \%$ & $3,3 \%$ \\
\hline 9 & & & $5,7 \%$ & $3,0 \%$ & $5,9 \%$ & $8,1 \%$ & $11,1 \%$ & $16,5 \%$ & $8,4 \%$ \\
\hline 10 & & $1,5 \%$ & & & & & & & $1,5 \%$ \\
\hline 11 & & $2,5 \%$ & & & & & & & $2,5 \%$ \\
\hline 12 & $6,7 \%$ & & & & $8,8 \%$ & $15,8 \%$ & $18,8 \%$ & $23,0 \%$ & $14,6 \%$ \\
\hline 13 & $0,3 \%$ & & & & & & & & $0,3 \%$ \\
\hline 14 & $9,7 \%$ & $9,8 \%$ & $7,3 \%$ & $6,5 \%$ & $4,3 \%$ & $2,1 \%$ & $3,2 \%$ & $1,3 \%$ & $5,5 \%$ \\
\hline 15 & $8,4 \%$ & $1,9 \%$ & & & & & $1,1 \%$ & $2,0 \%$ & $3,4 \%$ \\
\hline 16 & $12,9 \%$ & $6,6 \%$ & $14,2 \%$ & $12,5 \%$ & $10,0 \%$ & $11,2 \%$ & $14,7 \%$ & $7,5 \%$ & $11,2 \%$ \\
\hline 17 & & & $0,2 \%$ & & & & $0,8 \%$ & $2,3 \%$ & $1,1 \%$ \\
\hline 18 & & $9,5 \%$ & $17,9 \%$ & $13,1 \%$ & $12,7 \%$ & $7,1 \%$ & $9,3 \%$ & $4,3 \%$ & $10,6 \%$ \\
\hline 19 & $1,5 \%$ & $1,9 \%$ & & & & & & & $1,7 \%$ \\
\hline 20 & & $1,7 \%$ & $5,0 \%$ & & & & & & $3,4 \%$ \\
\hline 21 & & & $11,1 \%$ & $18 \%$ & $10,0 \%$ & $16,6 \%$ & $0,9 \%$ & & $11,3 \%$ \\
\hline 22 & $12,1 \%$ & $1,8 \%$ & & & & & & & $7,0 \%$ \\
\hline & & & & & & & & & \\
\hline
\end{tabular}

Com o objetivo de comparação entre a concentração de alocação de ativos nos portfólios formados pelos modelos $P E R$ e $M K W$ nesses 8 períodos, é disponibilizado pela Tabela 3 os portfólios formados por $M K W$, com os ativos e suas respectivas participações. Os portfólios formados pelo modelo $M K W$, como comentado na literatura, apresenta uma concentração maior na participação de alguns ativos. Para ilustrar essa situação atenta-se para os portfólios 7 e 8, as proporções de investimento no ativo de código 16 ultrapassam uma margem de $40 \%$, que na prática seria um resultado contestado pela questão de diversificação de carteiras. 
Tabela 3 - Portfólios formados e as respectivas participações dos ativos pelo modelo MKW

\begin{tabular}{|c|c|c|c|c|c|c|c|c|c|}
\hline $\begin{array}{c}\text { Cód. } \\
\text { Ativo }\end{array}$ & $\mathrm{P} 1$ & $\mathrm{P} 2$ & $\mathrm{P} 3$ & $\mathrm{P} 4$ & $\mathrm{P} 5$ & $\mathrm{P} 6$ & $\mathrm{P} 7$ & $\mathrm{P} 8$ & Média \\
\hline 1 & $8,3 \%$ & $14,8 \%$ & $17,4 \%$ & $15,9 \%$ & $15,9 \%$ & $6,1 \%$ & $6,2 \%$ & $13,8 \%$ & $12,3 \%$ \\
\hline 2 & $5,9 \%$ & $7,3 \%$ & $7,0 \%$ & $3,8 \%$ & $4,9 \%$ & $8,1 \%$ & $5,6 \%$ & $5,1 \%$ & $6,0 \%$ \\
\hline 3 & $11,6 \%$ & $7,5 \%$ & $6,1 \%$ & $7,2 \%$ & $6,8 \%$ & $6,7 \%$ & $4,4 \%$ & $2,9 \%$ & $6,6 \%$ \\
\hline 4 & $8,9 \%$ & $12,5 \%$ & $7,2 \%$ & $5,4 \%$ & $7,7 \%$ & $5,1 \%$ & $8,6 \%$ & $11,1 \%$ & $8,3 \%$ \\
\hline 5 & $4,7 \%$ & $4,8 \%$ & $1,0 \%$ & $3,8 \%$ & $2,6 \%$ & $6,3 \%$ & $3,8 \%$ & $3,3 \%$ & $3,8 \%$ \\
\hline 6 & $18,3 \%$ & $17,1 \%$ & $11,8 \%$ & $10,9 \%$ & $13,0 \%$ & $5,3 \%$ & $5,3 \%$ & $6,7 \%$ & $11,0 \%$ \\
\hline 7 & $4,4 \%$ & $3,3 \%$ & $1,4 \%$ & $0,5 \%$ & & $0,0 \%$ & & & $1,9 \%$ \\
\hline 8 & & & & & $0,8 \%$ & $2,4 \%$ & $2,0 \%$ & & $1,7 \%$ \\
\hline 9 & & & & & & $4,9 \%$ & $2,4 \%$ & $2,8 \%$ & $3,4 \%$ \\
\hline 10 & $5,7 \%$ & $4,6 \%$ & $3,4 \%$ & $3,4 \%$ & $2,8 \%$ & & $0,8 \%$ & $0,3 \%$ & $3,0 \%$ \\
\hline 11 & & & & & & & $1,1 \%$ & $0,7 \%$ & $0,9 \%$ \\
\hline 12 & $1,0 \%$ & $0,7 \%$ & $10,2 \%$ & $12,8 \%$ & $14,9 \%$ & $14,5 \%$ & $11,9 \%$ & $10,4 \%$ & $9,5 \%$ \\
\hline 13 & $5,7 \%$ & $2,7 \%$ & $9,3 \%$ & $8,2 \%$ & $6,9 \%$ & $3,1 \%$ & & & $6,0 \%$ \\
\hline 14 & & & & & & $1,1 \%$ & & & $1,1 \%$ \\
\hline 15 & $4,2 \%$ & & & & & & & & $4,2 \%$ \\
\hline 16 & $21,3 \%$ & $22,7 \%$ & $22,8 \%$ & $26,0 \%$ & $24,7 \%$ & $38,1 \%$ & $47,6 \%$ & $41,9 \%$ & $30,6 \%$ \\
\hline 17 & & $2,1 \%$ & $2,5 \%$ & $2,0 \%$ & $0,1 \%$ & & & & $1,7 \%$ \\
\hline
\end{tabular}

O desempenho das previsões obtidas pelos portfólios formados com o modelo PER ao longo de 12 e 24 semanas posteriores à otimização é apresentado pela Tabela 4 , onde representa o retorno do portfólio, $\theta$ a volatilidade (risco) e $S R$ o índice Sharpe no período, respectivamente. Considerando o reduzido número de portfólios obtidos pelo modelo PER não é possível afirmar se o mesmo desempenha melhor para prazos mais curtos ou longos de investimento. Adicionalmente, como os períodos de tempo se sobrepõem, não é possível concluir sobre os parâmetros conjuntamente. Todas as janelas de tempo de 52 semanas tem uma diferença de 3 semanas do próximo período, tanto no limite inferior quanto no superior, porém grande parte dos dados continua sendo a mesma. Atentando a este fato, se faz necessária a conclusão sobre estes parâmetros separadamente. Para o índice Sharpe, que maximiza a relação entre retorno e volatilidade, conclui-se que a magnitude dos valores deste parâmetro quando aplicado para uma previsão de 24 semanas é, em geral, superior. Isso se deve ao fato de que a magnitude dos valores dos retornos do portfólio também serem altos, em geral, em um prazo maior.

Tabela 4 - Valores dos parâmetros dos portfólios formados pelo modelo PER

\begin{tabular}{|c|c|c|c|c|c|c|c|}
\hline \multicolumn{3}{|c|}{12 semanas } \\
Período & $\mathrm{R}$ & $\theta$ & $\mathrm{SR}$ & Período & $\mathrm{R}$ & $\theta$ & $\mathrm{SR}$ \\
\hline $151-163$ & $5,26 \%$ & $2,73 \%$ & 1,93 & $151-175$ & $-2,77 \%$ & $2,39 \%$ & $-1,16$ \\
\hline $154-166$ & $1,30 \%$ & $2,55 \%$ & 0,51 & $154-178$ & $-6,50 \%$ & $2,23 \%$ & $-2,91$ \\
\hline $157-169$ & $-2,62 \%$ & $2,12 \%$ & $-1,24$ & $157-181$ & $2,21 \%$ & $2,04 \%$ & 1,08 \\
\hline $160-172$ & $-4,25 \%$ & $1,77 \%$ & $-2,39$ & $160-184$ & $-0,37 \%$ & $1,81 \%$ & $-0,2$ \\
\hline $163-175$ & $-1,22 \%$ & $1,57 \%$ & $-0,78$ & $163-187$ & $12,11 \%$ & $1,69 \%$ & 7,17 \\
\hline $166-178$ & $-0,94 \%$ & $1,76 \%$ & $-0,53$ & $166-190$ & $11,11 \%$ & $1,99 \%$ & 5,59 \\
\hline $169-181$ & $6,12 \%$ & $2,24 \%$ & 2,73 & $169-193$ & $14,07 \%$ & $2,28 \%$ & 6,16 \\
\hline $172-184$ & $1,74 \%$ & $2,69 \%$ & 0,65 & $172-196$ & $21,47 \%$ & $2,73 \%$ & 7,86 \\
\hline
\end{tabular}


Acredita-se que é possível para fins de comparação entre os modelos $P E R$ e $M K W$, estudados neste trabalho, verificar os respectivos desempenhos das previsões obtidas para o mercado brasileiro de ações em relação aos benchmarks de mercado. Essa comparação se dá entre a média dos parâmetros dos mesmos. Porém, é inviável a conclusão sobre as médias dos parâmetrosde cada modelo isoladamente, visto que os períodos estudados se sobrepõem. Os resultados podem ser observados na Tabela 5 onde representa a média dos retornos, a média da volatilidade (risco) e a média do índice Sharpe.

Tabela 5 - Média dos parâmetros de desempenho nas oito semanas

\begin{tabular}{|c|c|c|c|c|c|c|}
\cline { 2 - 7 } \multicolumn{1}{c|}{} & \multicolumn{3}{c|}{12 Semanas } & \multicolumn{3}{c|}{24 Semanas } \\
\cline { 2 - 7 } \multicolumn{1}{c|}{} & $R$ & $\bar{\theta}$ & $\overline{S R}$ & $\bar{R}$ & $\bar{\theta}$ & $\overline{S R}$ \\
\hline PER & $0,67 \%$ & $2,18 \%$ & 0,11 & $6,42 \%$ & $2,15 \%$ & 2,95 \\
\hline MKW & $0,19 \%$ & $1,78 \%$ & $-0,1$ & $5,16 \%$ & $1,76 \%$ & 3,02 \\
\hline IBOV & $-3,21 \%$ & $2,92 \%$ & $-1,02$ & $-3,47 \%$ & $2,99 \%$ & $-1,17$ \\
\hline IBRX-50 & $-3,82 \%$ & $2,88 \%$ & $-1,26$ & $-5,16 \%$ & $2,99 \%$ & $-1,75$ \\
\hline
\end{tabular}

Para os três parâmetros, são apresentadas na Tabela 5 suas respectivas médias ao longo desses 8 períodos. Quanto maior o retorno médio, melhor o desempenho da carteira. A volatilidade, entretanto, deve ser interpretada da forma contrária, visto que quanto maior seu valor, maior a chance de não se obter o retorno. O índice Sharpe deve ser interpretado de maneira análoga ao retorno, pois se tratando de um coeficiente de variação, quanto maior o seu valor, maior será o retorno e menor será a volatilidade do portfólio.
O modelo PER é superior aos IBRX-50 e IBOVESPA nas três métricas avaliadas e também superior ao $M K W$ com relação ao retorno. No entanto, ele é inferior ao $M K W$ na comparação do risco e índice Sharpe para 24 semanas. $\mathrm{O}$ modelo $M K W$ encontra soluções viáveis nos 64 períodos estudados. Com isto realizou-se a mesma sistemática descrita na seção 4.2 e se analisao desempenho do modelo $M K W$ nas previsões para $12 \mathrm{e}$ 24 semanas posteriores à otimização. Os resultados da Tabela 6 apresentam novamente a média dos retornos $\bar{R}$, a média da volatilidade (risco) $\bar{\theta}$ e a média do índice Sharpe $\overline{S R}$ para os 64 períodos.

Tabela 6 - Resultados do modelo MKW para 64 períodos

\begin{tabular}{|c|c|c|c|c|c|c|}
\cline { 2 - 7 } \multicolumn{1}{c|}{} & \multicolumn{3}{c|}{12 Semanas } & \multicolumn{3}{c|}{ 24 Semanas } \\
\cline { 2 - 7 } \multicolumn{1}{c|}{} & $\bar{R}$ & $\bar{\theta}$ & $\overline{S R}$ & $\bar{R}$ & $\bar{\theta}$ & $\overline{S R}$ \\
\hline MKW & $2,60 \%$ & $2,90 \%$ & 2,12 & $5,30 \%$ & $3,10 \%$ & 4,14 \\
\hline IBRX-50 & $0,48 \%$ & $3,73 \%$ & 0,38 & $1,50 \%$ & $3,95 \%$ & 1,14 \\
\hline IBOV & $0,95 \%$ & $3,61 \%$ & 0,57 & $2,70 \%$ & $3,80 \%$ & 1,47 \\
\hline
\end{tabular}

Conclui-se que a média dos retornos dos portfóliosformados pelo modelo $M K W$ nos 64 períodos estudados são superiores aos índices de benchmark. O modelo $M K W$ selecionou portfólios com melhor rentabilidade e menor exposição ao risco em relação aos benchmarkings selecionados. No entanto, o $M K W$ acaba gerando portfólios muito concentrados e não fornece nenhum indicativo de entrada e saída de mercado. Em contrapartida, o modelo PER é bastante sensível a definição a definição dos parâmetros de retorno e probabilidade de obtenção do mesmo. O sucesso deste modelo da definição de um bom par destes valores. Contudo, esta é uma tarefa bastante difícil, pois ela é dependente dos ativos e do período considerado.

\section{Conclusões}

Este trabalho propôs um estudo focado na avaliação da aplicação prática dos modelos de seleção de carteira proposto por Markowitz (1952), denominado de $M K W$, e do modelo alternativo proposto por Bonami e Lejeune (2009) e reformulado por Filomena e Lejeune (2012), denominado de PER, para a obtenção de portfólios de ações apresentando bom compromisso entre rentabilidade e exposição ao risco. Estudou-se o desempenho destes modelos no mercado brasileiro de ações, utilizando o preço de fechamento semanal dos ativos que compuseram o IBRX-50 na data de 23 de Agosto de 2011. Para a comparação de performance entre os 
modelos com os índices de benchmarks, utilizaram-se as seguintes três métricas: retorno, volatilidade e índice Sharpe.

Além das limitações do modelo $M K W$ discutidas na literatura, ao se utilizar o modelo PER neste trabalho pode-se constatar também algumas de suas limitações, já que quando aplicado no mercado brasileiro de ações, este gerou respostas viáveis em $12,5 \%$ das vezes em que foi utilizado. Um dos problemas é que foi disponibilizada apenas uma classe de ativos, ações, e não foram incluídos ativos livres de risco, commodities e moedas. A inclusão dos mesmos na cesta de ativos permitira a obtenção de soluções viáveis em um número bem superior ao obtido nos cenários de teste. Outro fator que justifica o baixo número de soluções viáveis foi à escolha do valor de $p$ de 0,2 . A redução deste valor de geraria um maior sucesso na obtenção de soluções viáveis. O que não necessariamente é um benefício, já que perder-se-ia a informação de entrada e saída de mercado. $\mathrm{O}$ fato de se obter uma solução inviável pode fornecer uma possível indicação de saída ou diminuição de investimentos aos investidores quando usando o modelo para a definição de suas carteiras.

Uma das vantagens ao se utilizar o modelo PER é a de que a matriz de variância-covariância não necessita ser positiva-semidefinida (FILOMENA; LEJEUNE, 2012), isto é, não há problema caso o número de ativos disponibilizados para a otimização seja elevado. Este é um desafio enfrentado constantemente pelos gestores de portfólio, pois existe um grande número de ativos no mercado. Somente em uma das bolsas de valores de Nova Iorque (NYSE) existem mais de 2.000 empresas listadas. Outras vantagens são: (i) os portfólios formados pelo modelo contemplam questões práticas como diversificação, não alocando excessivamente capital em um único ativo como o de $M K W$; e (ii) as previsões com o modelo PER superam os índices de benchmark nos períodos analisados neste trabalho, mesmo considerando que os períodos para os quais o modelo PER realiza as previsões, abrangem o período de 20 de Novembro de 2009 a 1 de Outubro de 2010, isto é, grande parte de 2010, que foi um ano em que a Bovespa ficou com retorno baixo, de aproximadamente $0,06 \%$.

Outra grande vantagem do modelo PER é de que os portfólios formados por este ficam menos expostos ao risco de estimação dos parâmetros do retorno devido à inclusão da restrição probabilística, diminuindo a sensibilidade do portfólio quanto à estimação dos retornos esperados dos ativos. Qualquer erro nessa estimação poderá enviesar a solução do portfólio em direção a ativos para os quais o retorno esperado foi superestimado,formando portfólios ineficientes e arriscados (CHOPRA; ZIEMBA, 1993). Além do modelo $M K W$ estar extremamente exposto a esse risco, através do valor do parâmetro $p$ no modelo PER é possível diferenciar níveis de incerteza diferentes associados às estimativas introduzidas no modelo.

\section{Referências}

BAWA, V.; BROWN, S.; KLEIN, R. Estimation Risk and Optimal Portfolio Choice. North-Holland: Elsevier Science Ltd, 1979.

BROADIE, M. Computing efficient frontiers using estimated parameters. Annals of Operations Research, v. 45, n. 1, p. 21-58, Dec. 1993

BONAMI, P.; LEJEUNE, M. A. An exact solution approach for portfolio optimization problems under stochastic and integer constraints. Operations Research, v. 57, n. 3, p. 650-670, May-June 2009.

CERIA, S.; STUBBS, R. A. Incorporating estimation errors into portfolio selection: Portfolio construction. Journal of Asset Management, v. 7, n. 2, p. 109-127, Jul. 2006.

CHOPRA, V.; ZIEMBA, W. T. The effects of errors in means, variances, and covariances on optimal portfolio choice. Journal of Portfolio Management, v. 19, n. 2, p. 6-11, Winter. 1993.

CORAZZA, M.; FAVARETTO, D. On the existence of solutions to the quadratic mixed-integer mean-variance portfolio selection problem. European Journal of Operational Research, v. 176, n. 3, p. 1947-1960, Feb. 2007.

CORNUEJÓLS, G.; TÜTÜNCÜ, R. Optimization methods in finance. Cambridge: Cambridge University Press, 2007.

DENTCHEVA, D.; RUSZCZYNSKI, A. Portfolio optimization with stochastic dominance constraints. Journal of Banking and Finance, v. 30, n. 2, p. 433451, Feb. 2006.

FILOMENA, T. P.; LEJEUNE, M. A. Stochastic Portfolio Optimization with Proportional Transaction Costs: Convex Reformulations and Computational Experiments. Operations Research Letters, v. 40, n. 3, p. 212-217, May 2012.

FILOMENA, T. P.; LEJEUNE, M. A. Warm-Start Heuristic for Stochastic Portfolio Optimization with Fixed and Proportional Transaction Costs. Working paper.

LI, D.; SUN, T. F.; WANG, J. Optimal lot solution to cardinality constrained mean-variance formulation for portfolio selection. Mathematical Finance, v. 16, n. 1, p. 83-101, Jan. 2006. 
LUENBERGER, D. G. Investment Science. Oxford: Oxford University Press, 1998. 494p.

MARKOWITZ, H. M. Portfolio section. Journal of Finance, v. 7, n. 1, p. 77-91, Mar. 1952.

MERTON, R. C. On estimating the expected return on the market: An exploratory investigation. Journal of Financial Economics, v. 8, n. 4, p. 323-361, Dec. 1980.

ROY, A. D. Safety first and the holdin assets. Econometrica, v. 20, n. 3, p. 431-449, Jul. 1952.

SCHERER. B.; MARTIN, D. Introduction to Modern Portfolio Optimization with NuOPT, S-PLUS and S+Bayes. New York: Springer, 2005.

SHARPE, W. Mutual fund performance. Journal of Business. v. 39, n. 1, p. 119-138, Jan. 1966.

TÜTÜNCÜ, R.; KOENIG, M. Robust asset alocation. Annals of Operations Research, v. 132, n. 1-4, p. 157-187, Nov. 2004.

ZENIOS, S. A. Practical Financial Optimization. Massachusests: Blackwell Publishing, 2008.

Recebido em 25/12/2011. Aceito em 31/07/2012. 